Standesangelegenheiten.

\title{
Zur Frage des Unterrichts in der Geschichte der Medizin an den Universitäten.
}

Von Dr. W. Haberling,

Dozent für Geschichte der Medizin in Düsseldorf.

Bei der Durchsicht der vielen Veröffentlichungen über die Neuordnung des Universitätsstudiums der Mediziner muß auffallen, daß nur selten des Unterrichts in der Geschichte der Medizin gedacht wird und daß kein Lehrplan auf diesen Zweig des Unterrichts Rücksicht nimmt. Es scheint also allgemein wie bisher die Uéberzeugung vorzuliegen, daß das Studium der Geschichte seines Standes für den Mediziner etwas Ueberflüssiges sei, dem er, wenn die übrigen Fächer ihn nicht vollkommen in Anspruch nehmen, zu seinem Vergnügen fröhnen kann, dem er aber nicht nachzugehen braucht, wenn er nicht will. Damit ist die Geschichte immer noch das Stiefkind in der großen Familie der Fächer, die der Studierende während seiner Studienzeit bewältigen muß, und es ist nur zu natürlich, daß der Student in erster Linie nur die Fächer belegt und hört, über die er im Examen Rechenschaft ablegen muB, und die Geschichte vollkommen vernachlässigt. Die Forderung der Deutschen Gesellschaft für die Geschichte der Medizin auf dem Wiener Naturforschertage 1913, dả auch für die Geschichte der Medizin das Hören eines Fachkollegs für die Studierenden der.Medizin vorgeschrieben werden möge, scheint ungehört verhallen $\mathrm{zu}$ sollen.

Dieser geringen Wertschätzung des Studiums der Geschichte entspricht auch das mangelnde Interesse des Staates und der medizinischen Fakultäten für die Besetzung von Lehrstühlen für die Geschichte Mer Mizin. 'Nur eine einzige deutsche Universität, Leipzig, hat eine ordentliche Honorarprofessur der Geschichte der Medizin, und selbst das unvergleichlich Große, das dort Sudhoff als freier Forscher seines Faches geleistet hat, hat noch keinen anderen Staat vermocht, diesem Beispiel zu folgen. Dagegen ist in Wien ebenfalls eine ordentliche Professur für Geschichte der Medizin mit Ne uburger besetzt. Im übrigen Deutschland aber müssen die anderen Dozenten der Geschichte neben ihrer Lehrtätigkeit, die ja ein ständiges, mühevolles, aber so dankbares und erfreuliches Studium fordert, noch irgendeinem anderen Broterwerb nacheehen der ihnen die schönsten Stunden ihrer Arbeitszeit für die Geschichte stiehlt. Aber noch mehr! Eine große Anzahl Universitäten entbehrt überhaupt des Lehrers der Geschichte, sodaß selbst ein wissensdurstiger Student auf ihnen die Sehnsucht nach diesem Studium nicht stillen kann. So haben sieben preuBische Universitäten: Breslau, Frankfurt a. M., Göttingen, Greifswald, Kiel, Köln, Marburg, keinen Lehrer der Geschichte, und nur drei : Berlin, Bonn, Königsberg und die Akademie in Düsseldorf sind mit Dozenten besetzt. Wesentlich günstiger ist Was Verhältnis bei den außerpreußischen Universitäten, in denen vier: Würzburg, Freiburg, Jena und München (bis vor kurzem auch Rostock) Lehrstühle für Geschichte haben, während fünf andere: Erlangen, Gießen, Hamburg, Heidelberg und Tübingen unbesetzt sind.

In einer Zeit, in der man allgemein darangeht, alte Ungerechtigkeiten und Ungereimtheiten aus der Welt zu schaffen, erscheint es eine dringende Forderung, daß die Aerztewelt für eine würdige Ausgestaltung des Unterrichts in der Geschichte ihres Standes Sorge trägt und zunächst bei den maßgebenden Stellen die Notwendigkeit betont, an allen Universitäten Lehrstuhle für das Fach der Geschichte der Heilkunde zu errichten. Wenn erst der Studierende verpflichtet wird, sich mit der Geschichte seines Standes zul beschäftigen, wird, darüber dürfte kein Zweifel herrschen, ér auch Lust und Liebe zu diesem Fach bekommen und die reichen Früchte ernten, die jeder sammelt, der nur einmal aus alten Zeiten heilende Kunst und heilende Künstler vor sich hat aufleben lassen.

Die Lehrer der Geschichte würden sicher eine Mehrbelastung des Lehrplans der Studierenden nicht befürworten, wenn sie nicht die Ueberzeugung aus ureigenster Erfahrung hätten, daß das Studium der Geschichte für den Studierenden eine Notwendigke it sei, daß ohne ihre Kenntnis der Arzt ein Fremdling in seinem Hause bleiben muß. Wer, wie der Verfasser, seit neun Jahren Gelegenheit gehabt hat, vor fertigen Aerzten in den Akademien Köln und Düsseldorf gelegentlich der Fortbildungskurse Vorlesungen über Geschichte zu halten, der weiß von vielen Aeußerungen unwilligen Erstaunens $\mathrm{zu}$ berichten, daß dem Studenten das ganze große Gebiet der Geschichte verschlossen geblieben ist, und mannigfache Nachfragen nach weiterer Ausbildungsmöglichkeit in diesem Fache zeugen davon, wie klar manchem Arzte es geworden ist, daß hier eine Lücke in seinem Wissen und in seiner Bildung klafft.

Warum halten wir nun das Studium der Medizingeschichte für so unbedingt notwendig? Weil es - um nur einiges aus immer wieder Gesagtem herauszugreifen - eine unumstößliche Tatsache ist, daß der, der die Vergangenheit einer Wissenschaft vernachlässigt, sie alle Tage neu beginnen muß. Es kann keinen Forscher im wahren Sinne des Wortes in irgendeinem Gebiete unserer Wissenschaft geben, der sich nicht davon uberzeugt, was vor ihm in seinem Forschungsgebiet geleistet worden 
ist. Jede neue Entdeckung ist ja immer das Resultat eines langen Entwicklungsprozesses, dessen leitende Idee in ihr zum Siege gebracht wird. Wo der historische Boden fehlt, da verdient das Resultat der Forschungen nicht, ein wissenschaftliches genannt zu werden. Wer diesen Grundsatz vernachlässigt und die Geschichte verachtet, an dem rächt sich diese Verachtung bitter. Unsere Zeit kennt ja nur zu gut jene dem planlosen Zufall vertrauenden Empiriker, die mit dünkelhafter Ueberlegenheit auf alles herabblicken, was nicht durch sie geschaffen ist. Unter ihnen gibt es nur zu viele, die den Charakter der Medizin ganz vergessen haben und nicht imstande sind, den Detailarbeiten, denen sie viel Zeit und Mühe geschenkt haben, den gebührenden Platz im Rahmen des Ganzen anzuweisen, sie in ihrer Bedeutung für die gemeinsame Wissenschaft zu würdigen. So wird mancher Arzt nur ein mechanischer Kunsthandwerker, nie ein zielbewußter Forscher und überschatzt zu leicht die Erfolge der Gegenwart. Vor dieser Gefahr bewahrt uns nur der Vergleich dessen, was die Gegenwart erzielt hat, mit dem, was die Vergangenheit an Errungenschaften hinterlassen hat. Den neu auftauchenden und wieder verschwindenden Lehrmeinungen und Behandlungsarten gegenüber können wir uns nur durch die Kenntnis der Geschichte ein sicheres Urteil bilden. "Die Geschichte ist es allein", betont Vetter, ,welche ermutigt "zum zweifelhaften Versuche, welche tröstet um das verfehlte Ziel, welche warnt vor den Folgen des Irrtums, der so oft, durch einen einzigen begangen, von Millionen gebüßt und verflucht wird. Sie, die Geschichte des Irrtums, ist die in der Schule des Unglücks erzogene Lehrerin der Wahrheit."

Und zum Sichlusse noch eins, das Wichtigste von allem! Sie führt uns das Ringen und Streben von. Tausenden und Abertausenden vor Augen, die Gesetze der allmächtigen Natur zu erforschen und die darüber erworbenen Kenntnisse zum Heile und Segen der leidenden Menschheit zu verwerten. Sie führt von den realistischen Beschäftigungen des ärztlichen Berufes am Krankenbett, im Laboratorium, am Seziertisch zu den idealen, humanitären Beschäftigungen und erfüllt das Herz des Studierenden mit edler Begeisterung für die Würde seines Standes und das Wohl der Menschheit.

Ueber die Art, wie der Unterricht in der Geschichte der Medizin erteilt werden soll, wird sich jeder Lehrer seine eigenen Ansichten gebildet haben. Nach meinem Dafürhalten kommt es vor allem darauf an, das In te res se des Studierenden zu erwecken und dauernd festzuhalten. Es ist nicht erforderlich, in dem Zeitraume von ein bis zwei Semestern den Lauf der Geschichte von den Uranfängen bis zum 19. Jahrhundert vor den Zuhörern abrollen zu lassen, vielmehr dürfte es weit ersprießlicher sein, an den Gipfelpunkten der Geschichte móglichst lange und ausführlich $\mathrm{zu}$ verweilen und bestimmte Zeiten hervorzuheben, als jedes Ereignis, auch das des speziellen Interesses bare, zu erwähnen. So genügte als Vorlesungsstoff für ein Semester vollauf die griechische Medizin oder noch besser das Zeitalter des Hippokrates, für ein anderes die Zeit der Renaissance, das 17. Jahrhundert usw. Um möglichst mannigfaltig das Interesse $\mathrm{zu}$ erregen, wären damit abzuwechseln Vorlesungen über Geschichte der Einzeldisziplinen, der Chirurgie, der Geburtshilfe, der Augenheilkunde, einzelner Krankheiten und Seuchen. Soviel wie möglich soll in diesen Vorlesungen die Zeit, die wir schildern, im Bilde vor uns wiedererstehen, die ärztliche Tätigkeit vor Augen gefuhrt werden und der Arzt selbst, den wir besprechen, mit seinen eigenen Worten gehört werden. Zahlreiche Lichtbilder sollen uns Heilstätten, Krankheiten, Verwundungen, anatomische Bilder, Heilmittel, die Gesichtszüge berühmter Aerzte, die Titel ihrer Werke vor Augen führen.

Nach meiner Ansicht soll der Studierende wenigstens einmal in einem vorklinischen und einmal in einem klinischen Semester die Geschichte der Medizin hören. Im vorklinischen Semester höre er die Geschichte seines Standes, die Geschichte der ärztlichen Ethik, die Geschichte der Anatomie und Physiologie; im klinischen die Vorlesungen, von denen ich oben gesprochen habe. Der Studierende sei sich dabei bewußt, daß das Gehorte ihn zum Studium der Geschichte an regen soll und daß er zur Vertiefung dieses Studiums noch in den persönlichen Arbeiten mit dem Lehrer Gelegenheit hat. In welcher Weise dieses Privatissimum einzusetzen hat, das hangt sowohl von den vorhandenen Mitteln, als auch von der Zahl zu weiteren Forschungen bereiter Studierender ab. So glückliche Verhältnisse wie in Leipzig, wo das Institut für Geschichte der Medizin mit seinen durch Sudhoff gesammelten Schätzen von Urkunden und Büchern dem Studierenden reichste Gelegenheit zur Fortbildung unter steter Führung des Meisters bietet, sind wohl in absehbarer Zeit an anderen Universitãten noch unerreichbar. Aber den einzelnen in einem historisch-medizinischen Seminar zu fördern, ihn bei der Auswahł einer geschichtlichen Dissertation tiefer in das genuBreiche Studium einzuführen, wird sich, wenn erst das Eis geschmolzen ist, welches den Quell des historischen Studiums solange hartnäckig verschlossen hat, überall durchführen lassen.

Zusammenfassung. Bei der Neuordnung des medizinischen Studiums ist dafür Sorge zu tragen, daß an jeder Universität Lehrstühle für besoldete Lehrer der Geschichte der Medizin eingerichtet werden und daB der Studierende verpflichtet wird, wenigstens in einem Semester Geschichte der Medizin zu hören. 